\title{
Are Serum Levels of Nuclear Factor Kappa B and Forkhead Box Protein P3 in Patients with Non-Alcoholic Fatty Liver Disease Related to Severity of Fibrosis?
}

\author{
Masoudreza Sohrabi ${ }^{1}$, Ali Gholami ${ }^{2,3}$,Bahareh AmirKalali ${ }^{1}$, Mahmoodreza Khoonsari ${ }^{1}$, \\ Roghieh Sahraei ${ }^{1}$, Mohsen NasiriToosi ${ }^{4}$,Farhad Zamani ${ }^{1}$,Hossein Keyvani 1,5,"
}

1. Gastrointestinal and Liver Disease Research Center (GILDRC), Iran University of Medical Sciences,Tehran, Iran

2. Noncommunicable Diseases Research Center, Neyshabur University of Medical Sciences, Neyshabur, Iran

3. Department of Epidemiology \& Biostatistics, School of Public Health, Neyshabur University of Medical Sciences, Neyshabur, Iran

4. Liver transplantation Research Center. Imam Khomeini Hospital, Tehran University of Medical Sciences.Tehran iran

5. Department of Virology, Iran University of Medical Sciences, Tehran, IR Iran

* Corresponding Author:

Hossein Keyvani, PhD

Department of Virology, Iran University of Medical Sciences, Tehran, IR Iran. Gastrointestinal and Liver Diseases Research Center, Iran University of Medical Sciences, Tehran, IR Iran.

Tel : + 982188941831

Fax : + 982188941831

Email: Keyvani.h@yahoo.com

Received : 29 Jan. 2021

Accepted : 02 Jun. 2021
ABSTRACT

\section{BACKGROUND}

Inflammation has a significant impact on the development and progression of fatty liver diseases.In this study, we aimed to investigate the relation between serum levels of nuclear factor kappa B (NFkB) and Forkhead box protein P3 (FOXP3)with fibrosis severity among patients with non-alcoholic fatty liver disease(NAFLD).

\section{METHODS}

In a prospective study, the patients suspicios of havingfatty liver were enrolled. The exclusion criteria lack of viral hepatitis, autoimmune hepatitis, Wilson's or other known liver diseases,history of liver or biliary surgery,bariatric surgery, and medications that influence liver metabolism. The participantsunderwent liver fibroscan.According to liver fibrosis, the patients weredivided into two groups; 1)fibrosis less than 7.2 KP,2)advanced NAFLD, fibrosis $\geq 7.3 \mathrm{KP}$. A10 cc fasting blood sample was taken from each patient for laboratory assessments. The variables between the two groups were compared using Chi-square or Fisher's exact test.The independence of cytokines was assessed by a logistic regression test.

\section{RESULTS}

Totally 90 patients were enrolled.The mean age was $42.21 \pm 11$ years. Of them, 50 and 47 participants were allocated to groups 1 and 2, respectively. In the univariate analysis, we revealed asignificant difference between age, body mass index (BMI), fasting blood glucose, liver enzymes, total cholesterol, andtriglyceride levels. Also, there was a significant difference betweenthe levels of NFKB and FOXP3 in group one compared with group two of the participants, as FOXP3(9.17 \pm 10.0 vs. $18.63 \pm 12.9 ; p<0.001)$ and NFKB $(1.70 \pm 1.70 ; p<0.01)$. After excluding the confounding factors, we observed a significant association between fibrosis level and cytokine levels in logistic regression.

\section{CONCLUSION}

Serum levels of NFKB and FOXP3 increased by advancing liver fibrosis in patients with NAFLD.This is an independent association. The identification of intermediary regulatory factors would be necessary

\section{KEYWORDS:}

NFKB, FOXP3, Liver,NAFLD, Fibrosis, Steatosis

Please cite this paper as:

Sohrabi MR, Gholami A, AmirKalali B, Khoonsari MR, Sahrae iR, NasiriToosi M, Zamani F , Keyvani H.Are Serum Levels of Nuclear Factor Kappa B and Forkhead Box Protein P3 in Patients with Non-Alcoholic Fatty Liver Disease Related to Severity of Fibrosis? Middle East J Dig Dis 2021;13: 356-362. doi: 10.34172/mejdd.2021.247. 


\section{INTRODUCTION}

Non-alcoholic fatty live disease(NAFLD)is a global health issue due to its vast morbidity and complications. In a large multiethnic cohort study, Setiawanand colleaguesconcluded that NAFLD was the main etiology of chronic liver disease across all ethnic groups and affected about $52 \%$ of participants. ${ }^{1}$

The pathophysiology of NAFLD, despite many attempts,remains unclear.It may be due to an imbalance of lifestyleas well as inflammation.It is considered that thepathogenesis of this disease follows multiple hit theories.In this theory,fat accumulation and inflammation arestrongly involved in NAFLD progression from simple steatosis to steatohepatitis,cirrhosis, and consequently liver cancer. ${ }^{2,3}$ The inflammation process that causes cell injury is developed by chronic. ${ }^{4,5,6,7}$

Cytokines and chemokinesregulate the function of inflammatory cells. Moreover, their production is strongly affected by the activation of transcription factors, including the nuclear factor kappa B (NFkB) and Forkhead box protein P3 (FOXP3). ${ }^{8} 10$

Studies showed that NFkB was primarily a cytoplasmic factor expressed by almost all types of cells and was a major inductive transcription factor that regulated a series of events at the molecular level that might be critical targets for treating inflammation. ${ }^{10-12}$ In normal and primary conditions, NFKB forms a complex in the cytoplasm of the unstimulated cells with its inhibitor, IкB. ${ }^{12,13} \mathrm{NFkB}$ activation can be induced upon physical (UV- or $\gamma$-irradiation), physiological (ischemia and hyperosmotic shock), or oxidative stresses. ${ }^{14}$ In pathological states such as the oxidant agents andthe presence of viral proteins, NFkB separates fromIKB, is transferred to the nucleus, and induces activation of various enzymes and proinflammatory molecules, including IL6 and TNF- $\alpha .3,{ }^{11}$

FOXP3 is also a key regulator of regulatory $\mathrm{T}$ (Treg) cell gene expression. Regulatory $\mathrm{T}$ cellsare heterogeneous $\mathrm{T}$ cells that play a crucial function in preserving peripheral immunological tolerance and control of immune responses toward pathogens and tumors. ${ }^{15}$ Also, former studies in humans illustrated that lack or muta- tions in the FOXP3 gene lead to autoimmune disordersand immune dysregulation. ${ }^{16,17}$

Studies in humansregarding the association between inflammatory factors and steatosis and fibrosis levels are limited. Indeed, non-invasive liver status evaluation can help us diagnose and assess the disease progression more simply. In a recent study by Monserrat-m and colleagues, intrahepatic fat content (IFC) measured by magnetic resonance imaging (MRI) was used to describe the fibrosis state and assessed the inflammatory factors according to IFC levels. They reported that the severity of NAFLD was associated with an increase in oxidative stress and proinflammatory status. ${ }^{18}$ Therefore according to the high prevalence of NAFLD and crucial roles of NFkB and FOXP3 in the development and progression of the disease,we designed a study to evaluate the association between NFKB and FOXP3 serum levels and liver fibrosis in a group of patients with NAFLD forfuture diagnostic and therapeutic purposes.

\section{MATERIALS AND METHODS}

\section{The study population}

This cross-sectional study was done among patients who had been referred to the liver clinic in Firoozgar Hospital from June 2017 to January 2019 for fatty liver assessment.According to laboratory scales, the inclusion criteria were adult patients aged more than 20 years with fatty liver in ultrasonography with or without elevated liver enzyme levels.Furthermore, subjects with normal to mildfatty liver in ultrasonography and normal liver enzymes were enrolled as the control group.

Exclusion criteria were viral hepatitis, autoimmune hepatitis, hepatic metabolic diseases, post-treatment of chronic hepatitis $\mathrm{C}$ infection, diabetes mellitus, bariatric surgery, taking medication with effects on liver status,or FOXP3 and NFKB levels such as silymarin or oral antidiabetics,anti-inflammatory medications, and alcohol consumption more than $30 \mathrm{~g} /$ day in men or more than $20 \mathrm{~g} /$ day in women. In the end, NAFLD was approved by an experienced gastroenterologist. 


\section{Definition of fatty liver}

\section{Ultrasonography}

The patients underwent ultrasonography by an expert radiologist. On ultrasonography, fatty liver is defined as normal, mild, and moderate to severe. A normal liver is defined when the consistency is homogeneous, with fine level echoes, minimally hyperechoic, or even isoechoic comparedwith the regular renal cortex..$^{19,20}$

\section{Fibroscan}

In the next step, the patients with approved fatty liver underwent fibroscanevaluation.In fact,fibroscan is a non-invasive method applying for assessment of liver stiffness measurement (LSM) and steatosis levelcontrol attenuated parameter (CAP). The fibroscanwas performed by an expert physicien in Firoozgar Hospital by using a fibroscan device (FibroScan; Echosens, Paris, France)with probes M and L. The examination was performed according to the standard protocol. ${ }^{15}$

\section{Laboratory assessments}

A $10 \mathrm{ml}$ of fasting venous blood was taken from each patient for laboratory assessment.An auto-analyzer BS200 (Mindray, Shenzhen, China) was used.In this context,biochemical examination including fasting blood sugar (FBS), total cholesterol (Chol), highdensity lipoprotein cholesterol (HDL-C), low-density lipoprotein cholesterol (LDL-C), triglycerides (TG), alanine aminotransferase (ALT), aspartate aminotransferase (AST), and alkaline phosphatase (ALP) were done by using Pars Azmoon Company (Pars Azmoon Co., Tehran, Iran) commercial diagnostic kits.

Human FOXP3 ELISA Kit (MBS2503897, Mybiosource Co., USA) was used for in vitro quantitative determination of human FOXP3 concentrations in serum by using a quantitative sandwich method according to the manufacturer's protocol. According to the protocol, ELISA kit for NFkB (MyBioSource, San Diego, California, United States) was used.

\section{Ethics}

In following Helsinki's declaration of medical ethics, this study was approved by the Ethics Committee of Iran University of Medical Sciences (ethical code IR.IUMS1397.32992). Written informed consent was obtained from each participant before enrollment.

\section{Data analysis}

Participants were categorized into two following groups according to fibrosis: 1) groupone; fibrosis less than $<7.2 \mathrm{KP}, 2$ )group 2 with advanced fibrosis $\geq 7.2$ kpand steatosis $\geq 290 \mathrm{dbm}$.

The descriptive data are presented as mean \pm SD. Chi-square or Fisher's exact test was used to evaluate differences between the groups. Univariate analysis andlogistic regression model were finally performed to evaluate the independent effects of NFKB and FOXP3 on liver fibrosis (age, BMI, liver enzymes, lipid profile, and FBS were considered as confounding factors). The data were analyzed using SPSS software version 20.0 (IBM-SPSS, IL, USA).P values lower than 0.05 were considered statistically significant.

\section{RESULTS}

Totally 97 patients were enrolled. 50 patients were categorized in group one with normal fibrosis, and 47 patients were categorized as having advanced fibrosis. The mean age was $42.0 \pm 11.30$ years. $60.0 \%$ of them were female. Table 1 shows the descriptive characteristics of the participants in the two groups of patients, including descriptive laboratory results. BMI, LDL, and HDL were not significantly different among the groups.

In the univariate analysis, we observed that the serum NFkB was significantly lower in group 1 than group $2(1.70 \pm 1.70$ vs. $3.52 \pm 2.39, p<0.001$ [95\%CI $=1.22-1.84]$. Regarding the FOXP3 level, we observed asignificant differencein FOXP3concentrations in group 1 vs. group2 $(9.10 \pm 9.90$ vs. $18.62 \pm 12.9, p<$ $0.001[95 \% \mathrm{CI}=1.03-1.11])($ table2).

In the next step, a multiple logistic regression model was applied. After adjustment of age,sex,BMI, liver enzymes, and lipid profile,we observed that the levels of NFkB and FOXP3 were not independently associated with advanced liver fibrosis (table 3 ).

\section{DISCUSSION}

In the present study, we showed that the serum levels of NFKB and FOXP3 hadsignificantly positive associations 
Table 1: Basic characteristic of participants according to fibrosis stages

\begin{tabular}{|c|c|c|c|c|}
\hline \multirow{2}{*}{ Variables } & \multicolumn{2}{|c|}{ Fibrosis status } & \multirow[t]{2}{*}{ Total $(\mathbf{N}=97)$} & \multirow[t]{2}{*}{$p$-value } \\
\hline & Normal $(\mathrm{N}=\mathbf{5 0})$ & Abnormal $(N=47)$ & & \\
\hline Age(year) & $37.2 \pm 10.0$ & $47.51 \pm 10.3$ & $42.2 \pm 11.3$ & 0.05 \\
\hline $\operatorname{Sex}(M / F)(N)$ & $22 / 28$ & $16 / 31$ & $38 / 59$ & 0.04 \\
\hline BMI & $26.7 \pm 6.12$ & $31.8 \pm 4.76$ & 32.70 & 0.08 \\
\hline WC & $97.6 \pm 14.3$ & $103.2 \pm 24.2$ & $103.3 \pm 19$ & 0.06 \\
\hline Wrist C & $18 \pm 5.1$ & $18.2 \pm 2.2$ & $18.01 \pm 4.0$ & 0.02 \\
\hline FBS & $95.4 \pm 16.9$ & $123.5 \pm 44.2$ & $107.9 \pm 34.8$ & 0.001 \\
\hline Total Chol & $168.3 \pm 46.6$ & $216.1 \pm 46.5$ & $189.6 \pm 52.1$ & 0.02 \\
\hline LDL & $114.7 \pm 33.4$ & $127.1 \pm 32.8$ & $120.2 \pm 35.5$ & 0.23 \\
\hline HDL & $43.01 \pm 9.2$ & $44.06 \pm 8.6$ & $43.7 \pm 8.9$ & 0.54 \\
\hline TG & $133.30 \pm 70.84$ & $194.7 \pm 99.2$ & $161.8 \pm 89.7$ & 0.01 \\
\hline AST & $22.40 \pm 10.4$ & $60.35 \pm 33.80$ & $40.7 \pm 31.1$ & 0.01 \\
\hline ALT & $25.5 \pm 25.30$ & $74.60 \pm 39.31$ & $48.8 \pm 41.2$ & 0.01 \\
\hline ALP & $160.30 \pm 54.70$ & $228.30 \pm 48.50$ & $193.2 \pm 61.8$ & 0.01 \\
\hline FOXP3 & $9.56 \pm 9.40$ & $17.5 \pm 13.0$ & $13.55 \pm 12.31$ & 0.001 \\
\hline NFkB & $1.84 \pm 1.7$ & $3.45 \pm 2.41$ & $2.54 \pm 2.23$ & 0.001 \\
\hline
\end{tabular}

BMI: Body mass index; WC: Waist circumference; FBS: Fasting blood sugar; Chol: Cholesterol; LDL: Low-density lipoprotein; HDL: High-density lipoprotein; TG: Triglyceride; AST: Aspartate transaminase; ALT: alanine aminotransferase; ALP: Alkaline phosphatase

Table 2: Univariate analysis of the association between studied factors and fibrosis

\begin{tabular}{lccc}
\hline Variables & OR $(\mathbf{9 5 C I})$ & Standard error & $p$-value \\
\hline Age $($ year $)$ & $1.100(1.05-1.15)$ & 0.025 & $<0.001$ \\
\hline Sex $(\mathrm{M} / \mathrm{F})(\mathrm{N})$ & $1.11(0.50-2.52)$ & 0.056 & $<0.001$ \\
\hline BMI & $1.21(1.12-1.33)$ & 0.056 & $<0.001$ \\
\hline FBS & $1.08(1.03-1.13)$ & 0.002 & $<0.001$ \\
\hline CHOL Total & $1.01(1.00-1.02)$ & 0.005 & 0.002 \\
\hline LDL & $1.00(099-1.03)$ & 0.007 & 0.243 \\
\hline HDL & $0.98(0.94-1.04)$ & 0.023 & 0.656 \\
\hline TG & $1.00(1.00-1.02)$ & 0.003 & 0.005 \\
\hline AST & $1.09(1.05-1.13)$ & 0.020 & $<0.001$ \\
\hline ALT & $1.10(1.05-1.13)$ & 0.018 & $<0.001$ \\
\hline ALP & $1.02(1.01-1.03)$ & 0.005 & $<0.001$ \\
\hline FOXP3 & $1.08(1.03-1.11)$ & 0.020 & $<0.001$ \\
\hline NFkB & $1.50(1.22-1.85)$ & 0.160 & $<0.001$ \\
\hline
\end{tabular}

OR: Odds ratio; CI: Confidence interval; BMI: Body mass index; WC: Waist circumference; FBS: Fasting blood sugar; Chol: Cholesterol;

LDL: Low-density lipoprotein; HDL: High-density lipoprotein; TG: Triglyceride; AST: Aspartate transaminase; ALT: alanine aminotransferase; ALP: Alkaline phosphatase

Table 3: Multiple analysis of the association between FOXP3 and NFKB levelsand fibrosis

\begin{tabular}{lccc}
\hline & OR $(\mathbf{9 5} \% \mathbf{C I})$ & Standard error & $p$-value \\
\hline FOXP3 & $1.37(0.730-2.620)$ & 0.450 & 0.320 \\
\hline NFkB & $13.07(0.075-22960.82)$ & 49.83 & 0.500 \\
\hline
\end{tabular}

OR: Odds ratio; CI: Confidence interval 
with the liverfibrosisstage along with liver enzymes and lipid profiles. Furthermore, Inthe multipleregression model,we did not observe independent relation of FOXP3 with liver fibrosis. However, NFkB with $p=0.50$ may havea weak independent role in the progress of fibrosis.

The pathogenesis of NAFLD ismultifaceted. In NAFLD,oxidative stress and lipotoxicity were seen secondary to lipid accumulation that consequently cause inflammatory responses, leading to alterations in inflammatory cytokines and eventually hepatocyte damage, including fibrosis development and its complications. ${ }^{21,22}$ Hence, inflammation hasbeen a notable concern in liver diseases. Itmight be a good research issuein preventing and treating this disease. In this regard, NFKB and FOXP3 may play essential roles. We observed that levels of NFKB and FOXP3 are associated with the fibrosis stage.Hence, we can consider that the inflammation process initiation may occur in the primary stage of NAFLD and liver fibrosis. Indeed,the progression of NAFLD seems to be dependent on different factors.

The NFkB has a significant role in the expression and regulation of proinflammatory substances, including chemokines and cytokines, andthe regulator of immune development and immune responses. ${ }^{10-12} \mathrm{In}$ the present study, in regression analysis, we observed a significant association between NFKB and severe fibrosis(table 2).NFKB is activated by different stress factors and has many liver pathologies, including NAFLD, alcoholic liver damage, and hepatic cancers, but the clinical implications were not elucidated. ${ }^{23}$ In the present study, we found that NFKBlevel isassociated withfibrosisstages, but the independent effect of NFKB needs more studies. Hence other confounding factors such as TG and cholesterol can play their roles independently or synergically with NFKB. Therefore we can consider that triggeringthe NFKB pathway may be complex and be initiated by different substances or circumstances.In fact, activation of NFKB is the first event in viral and non-viral liver diseases. Former studies demonstrated that this substance had dual functions; proinflammatory and antiapoptotic;therefore, imbalance of its concentration may lead to an inappropriate response that may cause expression of inflammation reactions.9,24 According to NFkB's vital role in hepatic injury and fibrosis,itshould be considered for a new therapeutic protocol in liver diseases. ${ }^{25,26}$

In the present study, results showed a positive association between FOXP3 serum level and liver fibrosis progression comparable with former reports.Matthaios and colleagues,in a study on liver specimens of patients with chronic liver diseases, revealed that expression of FOXP3 increased in the liver tissue, which was positively associated with inflammation severityindependent of its primary etiology. ${ }^{27}$ AmorasEda Sand coworkers, in their study on patients with chronic liver diseases, reported a high expression of FOXP3 along with advancing of diseases. ${ }^{28}$ In this context,Wie and others, in a study on regulatory $\mathrm{T}$ cell components indicated that FOXP3 positive cells might havean important function in limiting liver injury. ${ }^{29}$ Regulatory T-cells are a subtype of CD4 cells that work as inhibitors of effectors Tcell, NK cell, and consequently helping to maintain the inflammatory homeostasis. These cells, despite different types, express FOXP3, which is the major marker and functional balancing of regulatory $\mathrm{T}$ cell. The balance between effector and regulatory $\mathrm{T}$ cells causesthe elimination of viral hepatitis. ${ }^{30-32}$ Whether the role of FOXP3 in patients with NAFLD is the same has not been understood well.Furthermore, we illustrated the increasing level of FOXP3 by advancing fibrosis. It was observed that mRNA levels of the transcription factor FOXP3 were lower in groups of patients without fibrosis (stage F0) and increased by advancing fibrosis and inflammation regardless of the cause. ${ }^{28}$ Therefore, according to these studies, we can offer that ourfindings were in line with previous studies.In fact, we observed an imbalance of inflammatory regulation along with steatosis and advanced stages of fibrosis.As mentioned above, NFkB has a regulatory function, and FOXP3 is expressed as an anti-inflammatory substance. It can be consideredthat steatosis, as a milestone of NAFLD, can induce an inflammatory processthat may be presented by elevated liver enzymes and developing fibrosis. How the inflammatory regulatory system goes off balance and fails to protect the cell 
needs more studies.

\section{CONCLUSION}

The current study indicates that NFKB andFOXP3 serum levelssignificantly increase with the progress of NAFLD fibrosis levels. The increasing level of these factors indicates their key role, particularly the NFKB, in the progression of fibrosis. These effects may be applied in different pathways. Therefore other cofactors or intermittent substances would be considered.

\section{ETHICAL APPROVAL}

There is nothing to be declared.

\section{CONFLICT OF INTEREST}

The authors declare no conflict of interest related to this work.

\section{REFERENCES}

1. Setiawan VW, Stram DO, Porcel J, Lu SC, Le Marchand L, Noureddin M. Prevalence of chronic liver disease and cirrhosis by underlying cause in understudied ethnic groups: The multiethnic cohort. Hepatology 2016;64:1969-77. doi: 10.1002/hep. 28677

2. Petta S, Gastaldelli A, Rebelos E, Bugianesi E, Messa P, Miele L, et al. Pathophysiology of Non Alcoholic Fatty Liver Disease. Int J Mol Sci 2016;17:2082. doi: 10.3390/ ijms17122082

3. Gao B, Tsukamoto H. Inflammation in Alcoholic and Nonalcoholic Fatty Liver Disease: Friend or Foe? Gastroenterology 2016;150:1704-9. doi: 10.1053/j.gastro.2016.01.025

4. Sanyal AJ, Campbell-Sargent C, Mirshahi F, Rizzo WB, Contos MJ, Sterling RK, et al. Nonalcoholic steatohepatitis: association of insulin resistance and mitochondrial abnormalities. Gastroenterology 2001;120:1183-92. doi: 10.1053/ gast.2001.23256

5. Begriche K, Igoudjil A, Pessayre D, Fromenty B. Mitochondrial dysfunction in NASH: causes, consequences and possible means to prevent it. Mitochondrion 2006;6:1-28. doi: 10.1016/j.mito.2005.10.004

6. Sutti S, Jindal A, Locatelli I, Vacchiano M, Gigliotti L, Bozzola $\mathrm{C}$, et al. Adaptive immune responses triggered by oxidative stress contribute to hepatic inflammation in NASH. Hepatology 2014;59:886-97. doi: 10.1002/hep.26749

7. Zhang XQ, Xu CF, Yu CH, Chen WX, Li YM. Role of endoplasmic reticulum stress in the pathogenesis of nonalcoholic fatty liver disease. World J Gastroenterol 2014;20:1768-76. doi: 10.3748/wjg.v20.i7.1768

8. Lee YA, Wallace MC, Friedman SL. Pathobiology of liver fibrosis: a translational success story. Gut 2015;64:830-41. doi: 10.1136/gutjnl-2014-306842
9. Nisr RB, Shah DS, Ganley IG, Hundal HS. Proinflammatory NFkB signalling promotes mitochondrial dysfunction in skeletal muscle in response to cellular fuel overloading. Cell Mol Life Sci 2019;76:4887-4904. doi: 10.1007/s00018-01903148-8

10. Mitchell S, Vargas J. Signaling via the NFאB system. Wiley Interdiscip Rev Syst Biol Med 2016;8:227-41. doi: 10.1002/ wsbm.1331

11. Oeckinghaus A, Ghosh S. The NF-kappaB family of transcription factors and its regulation. Cold Spring Harb Perspect Biol 2009;1:a000034. doi: 10.1101/cshperspect.a000034.

12. Luedde T, Schwabe RF. NF- $\mathrm{kB}$ in the liver--linking injury, fibrosis and hepatocellular carcinoma. Nat Rev Gastroenterol Hepatol 2011;8:108-18. doi: 10.1038/nrgastro.2010.213.

13. Scheidereit C. IkappaB kinase complexes: gateways to NFkappaB activation and transcription. Oncogene 2006;25:6685705. doi: 10.1038/sj.onc. 1209934

14. Hayden MS, West AP, Ghosh S. NF-kappaB and the immune response. Oncogene 2006;25:6758-80. doi: 10.1038/ sj.onc. 1209943

15. Wong VW, Petta S, Hiriart JB, Cammà C, Wong GL, Marra $\mathrm{F}$, et al. Validity criteria for the diagnosis of fatty liver by $\mathrm{M}$ probe-based controlled attenuation parameter. $J$ Hepatol 2017;67:577-84. doi: 10.1016/j.jhep.2017.05.005

16. Georgiev P, Charbonnier LM, Chatila TA. Regulatory T Cells: the Many Faces of Foxp3. J Clin Immunol 2019;39:623-40. doi: 10.1007/s10875-019-00684-7

17. Jia H, Qi H, Gong Z, Yang S, Ren J, Liu Y, et al. The expression of FOXP3 and its role in human cancers. Biochim Biophys Acta Rev Cancer 2019;1871:170-178. doi: 10.1016/j. bbcan.2018.12.004

18. Monserrat-Mesquida M, Quetglas-Llabrés $\mathrm{M}$, Abbate $\mathrm{M}$, Montemayor S, Mascaró CM. Casares M , et al. Oxidative Stress and Proinflammatory Status in Patients with Non-Alcoholic Fatty Liver Disease. Antioxidants 2020;9: 759. doi: 10.3390/antiox9080759.

19. Lee SS, Park SH, Kim HJ, Kim SY, Kim MY, Kim DY, et al. Non-invasive assessment of hepatic steatosis: prospective comparison of the accuracy of imaging examinations. J Hepatol 2010;52:579-85. doi: 10.1016/j.jhep.2010.01.008

20. Mahale AR, Prabhu SD. Clinical relevance of reporting fatty liver on ultrasound in asymptomatic patients during routine health checkups. $J$ Int Med Res 2018;46:4447-54. doi: 10.1177/0300060518793039

21. Buzzetti E, Pinzani M, Tsochatzis EA. The multiple-hit pathogenesis of non-alcoholic fatty liver disease (NAFLD). Metabolism 2016;65:1038-48. doi: 10.1016/j.metabol.2015.12.012

22. Anty R, Gual P. Pathogenesis of non-alcoholic fatty liver disease. Presse Med 2019;48:1468-83. doi: 10.1016/j. lpm.2019.09.051.

23. Khullar M, Sharma A, Wani A, Sharma N, Sharma N, Chandan BK, et al. Acteoside ameliorates inflammatory responses through NFkB pathway in alcohol induced hepatic damage. Int Immunopharmacol 2019;69:109-117. doi: 10.1016/j.in- 
timp.2019.01.020.

24. Liu T, Zhang L, Joo D, Sun SC. NF- $\mathrm{B}$ signaling in inflammation. Signal Transduct Target Ther 2017;2:17023. doi: 10.1038/sigtrans.2017.23. 10.1038/sigtrans.2017.23

25. Sunami Y, Ringelhan M, Kokai E, Lu M, O’Connor T, Lorentzen A, et al. Canonical NF- $\mathrm{BB}$ signaling in hepatocytes acts as a tumor-suppressor in hepatitis B virus surface antigen-driven hepatocellular carcinoma by controlling the unfolded protein response. Hepatology 2016;63:1592-607. doi: 10.1002/hep.28435

26. Nisr RB, Shah DS, Ganley IG, Hundal HS. Proinflammatory NFkB signalling promotes mitochondrial dysfunction in skeletal muscle in response to cellular fuel overloading. Cell Mol Life Sci 2019;76:4887-4904. doi: 10.1007/s00018-01903148-8.

27. Speletas M, Argentou N, Germanidis G, Vasiliadis T, Mantzoukis K, Patsiaoura K, et al. Foxp3 expression in liver correlates with the degree but not the cause of inflammation. Mediators Inflamm 2011;2011:827565. doi: 10.1155/2011/827565.

28. Amoras Eda S, Gomes ST, Freitas FB, Santana BB, Ishak G, Ferreira de Araújo MT, et al. Intrahepatic mRNA Expression of FAS, FASL, and FOXP3 Genes Is Associated with the Pathophysiology of Chronic HCV Infection. PloS One 2016;11:e156604. doi: 10.1371/journal.pone.0156604

29. Wei HX, Chuang YH, Li B, Wei H, Sun R, Moritoki Y, et al. CD4+ CD25+ Foxp3+ regulatory $\mathrm{T}$ cells protect against $\mathrm{T}$ cell-mediated fulminant hepatitis in a TGF-beta-dependent manner in mice. J Immunol 2008;181:7221-9. doi: 10.4049/ jimmunol.181.10.7221

30. Kanamori M, Nakatsukasa H, Okada M, Lu Q, Yoshimura A. Induced Regulatory T Cells: Their Development, Stability, and Applications. Trends Immunol 2016;37:803-11. doi: 10.1016/j.it.2016.08.012

31. Wing JB, Tanaka A, Sakaguchi S. Human FOXP3(+) Regulatory $\mathrm{T}$ Cell Heterogeneity and Function in Autoimmunity and Cancer. Immunity 2019;50:302-16. doi: 10.1016/j.immuni.2019.01.020

32. Alatrakchi N, Koziel M. Regulatory T cells and viral liver disease. J Viral Hepat 2009;16:223-9. doi: 10.1111/j.13652893.2009.01081. 